\title{
Organic light emitting bistable memory device with high on/off ratio and low driving voltage
}

\author{
Sung Hyun Kim, ${ }^{1}$ Kyoung Soo Yook, ${ }^{2}$ Jun Yeob Lee, ${ }^{2, a)}$ and Jyongsik Jang ${ }^{1}$ \\ ${ }^{1}$ School of Chemical and Biological Engineering, Seoul National University, Shinlim-dong, Kwanak-gu, \\ Seoul 151-742 Republic of Korea \\ ${ }^{2}$ Department of Polymer Science and Engineering, Dankook University, Jukjeon-dong, Suji-gu, Yongin-si, \\ Kyeonggi-do 448-701, Republic of Korea
}

(Received 14 April 2008; accepted 2 July 2008; published online 8 August 2008)

\begin{abstract}
Organic light emitting bistable memory devices (OLEBDs) with a dual function of organic light emitting diodes and organic memory devices were developed by using $0.5 \mathrm{~nm}$ thick $\mathrm{MoO}_{3}$ as an interlayer between hole injection layer and hole transport layer. The hole transport unit with $\mathrm{MoO}_{3}$ interlayer played a role of a memory unit as well as a hole transport unit. High on/off ratio over 1000 was obtained at a reading voltage of $1 \mathrm{~V}$ and driving voltage was lowered by $\mathrm{MoO}_{3}$. In addition, two different luminances were obtained at the same driving voltage by changing writing voltage of OLEBDs. (C) 2008 American Institute of Physics. [DOI: 10.1063/1.2964178]
\end{abstract}

Organic bistable memory devices (OBDs) have been studied intensively due to their merits of simple device structure, simple fabrication process, and design versatility. ${ }^{1-14}$ They can be fabricated into a fully flexible shape and merged into flexible electronic systems. ${ }^{1}$

There have been many studies about OBDs and most of works were focused on developing OBDs with a high on/off ratio and a long-term stability. Metal nanoparticles (NPs) were found to be effective to get stable memory characteristics and many different metal NPs were reported to improve the memory performances of OBDs. ${ }^{2-9}$ Pure organic memory devices without metal NPs were also studied and a molecularly doped organic system could play a role of OBDs. ${ }^{10-16}$ Some polymers such as polyfluorene and polyphenylenevinylene (PPV) could work as an active material in OBDs. ${ }^{15,16}$ However, memory performances were not as good as those of metal NP based OBDs.

In addition to OBDs, an OBD with a dual function of memory and light-emitting devices was introduced by Tseng et al. ${ }^{17}$ It had a light-emitting unit interfaced with OBD unit containing Au NPs and it could function as a memory device as well as a light emitting device. However, light-emitting performances were not good enough as a light-emitting device because organic memory unit was not effective as a charge transport unit. Other than this, PPV based organic light emitting diodes (OLEDs) showed bistable memory performances ${ }^{15}$ and common OLEDs also exhibited bistability even though detailed mechanism for the bistability was not clarified. ${ }^{18,19}$

In this work, organic light emitting bistable memory devices (OLEBDs) with a dual function of a nonvolatile memory and light-emitting devices were developed by using $\mathrm{MoO}_{3}$ as an interlayer between a hole injection layer (HIL) and a hole transport layer (HTL). Device performances of OLEBDs were investigated both as a memory device and light-emitting devices.

A device configuration for the OLEBDs was indium tin oxide $(150 \mathrm{~nm}) / N, N^{\prime}$-diphenyl- $N, N^{\prime}$-bis-[4-(phenyl-m-

\footnotetext{
${ }^{\text {a) }}$ Author to whom correspondence should be addressed. Tel: 82-31-80053585. FAX: 82-31-8005-3585. Electronic mail: leej17@dankook.ac.kr.
}

tolyl-amino)-phenyl]-biphenyl $-4,4^{\prime}$-diamine (DNTPD, 60 $\mathrm{nm}) / \mathrm{MoO}_{3}(x \mathrm{~nm}) / \mathrm{N}, \quad \mathrm{N}$ '-di(1-naphthyl)-N,N'-diphenylbenzidine (NPB, $30 \mathrm{~nm}) /$ tris (8-hydroxyquinoline) aluminum $\left(\mathrm{Alq}_{3}, 20 \mathrm{~nm}\right) / \mathrm{LiF}(1 \mathrm{~nm}) / \mathrm{Al}(200 \mathrm{~nm})$. Thickness of $\mathrm{MoO}_{3}$ was 0 and $0.5 \mathrm{~nm}$. DNTPD was a HIL and NPB was used as a HTL. $\mathrm{Alq}_{3}$ was an emitting material as well as an electron transport material. All organic materials were deposited at a deposition rate of $1 \AA$ s and $\mathrm{MoO}_{3}$ was evaporated at a evaporation rate of $0.1 \AA / \mathrm{s}$. Morphology of $\mathrm{MoO}_{3}$ on DNTPD was observed with transmission electron microscope (TEM) (Jeol) and current-voltage-luminance $(I-V-L)$ characteristics of the devices were measured with Keithley 2400 source measurement unit and PR 650 spectrophotometer.

The merit of using OLEBDs is that we can obtain different luminances at the same voltage. In general, the luminance is controlled by a thin film transistor, but it can be managed by just changing the writing voltage of bistable light emitting devices. Therefore, it is possible to fabricate self-driven OLEDs using OLEBDs without any driving circuit. In addition, driving voltage can be lowered because high luminance can be obtained at the same voltage after switching on the device.

$\mathrm{MoO}_{3}$ has been known to form a charge transfer (CT) complex with aromatic amine materials and it could be used as a $p$-type dopant in OLEDs. ${ }^{20}$ It can also be used as an inorganic NP to trap charges in organic matrix and can induce a bistable current level in OBDs. ${ }^{21}$ Therefore, $\mathrm{MoO}_{3}$ interlayer can act as an active layer for OBDs and a HTL in OLEDs.

A uniform dispersion of NPs in organic matrix is important to get good performances as OBDs and OLEDs because aggregation of NPs may lead to unstable device performances and a poor reproducibility. Therefore, the morphology of $\mathrm{MoO}_{3}$ interlayer on DNTPD was investigated. Figure 1 shows a TEM picture of $\mathrm{MoO}_{3}$ deposited on the DNTPD layer. $\mathrm{MoO}_{3}$ was uniformly dispersed on the DNTPD layer and the particle size of $\mathrm{MoO}_{3}$ was ranged from 10 to $100 \mathrm{~nm}$ with an average particle size of $80 \mathrm{~nm}$. No extensive aggregation of NPs was observed and the morphology was quite stable even after several weeks. 


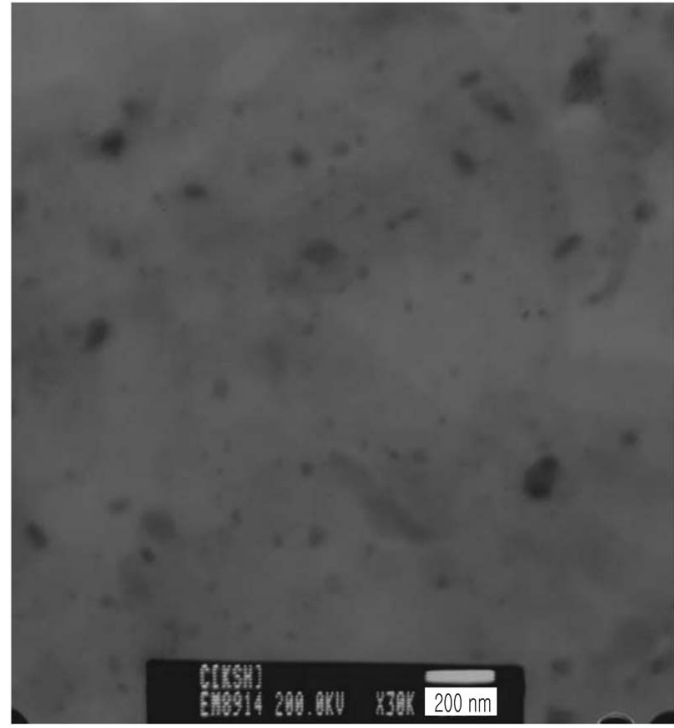

FIG. 1. TEM pictures of $0.5 \mathrm{~nm}$ thick $\mathrm{MoO}_{3}$ interlayer on hole injection layer.

OLEDBs fabricated in this work have a dual function of OBDs and OLEDs at the same time and OBD characteristics of the OLEBDs were measured. Figure 2 shows $I$ - $V$ curves of the OLEBDs with $\mathrm{MoO}_{3}$ interlayer. A standard device without $\mathrm{MoO}_{3}$ interlayer did not show any memory behavior. Compared with the standard device, $0.5 \mathrm{~nm}$ thick $\mathrm{MoO}_{3}$ interlayer device showed memory characteristics during forward and reverse scans. A low conductivity state (LCS) was observed from 0 to $2.3 \mathrm{~V}$ in forward scan and a sharp increase of current level from $10^{-7}$ order to $10^{-4} \mathrm{~A}$ order was detected at a turn-on voltage of $2.3 \mathrm{~V}$. The LCS was changed into high conductance state (HCS) at $2.3 \mathrm{~V}$. The HCS was maintained during reverse scan and a high current was obtained between 0 and $2.3 \mathrm{~V}$. There was a large difference of current level between the LCS and the HCS in $0.5 \mathrm{~nm}$ thick $\mathrm{MoO}_{3}$ device and the on/off ratio was 1000 . In the reverse scan of memory devices, the HCS was stable up to $-2.2 \mathrm{~V}$ and the current level abruptly decreased from $-2.3 \mathrm{~V}$. The low current level in reverse voltage corresponds to the LCS of OBDs and the on/off cycle could be stably operated many times. A write and erase voltage could be set to 4 and $-4 \mathrm{~V}$, respectively, and the on/off ratio at a reading voltage of $1 \mathrm{~V}$ was 1000 in $0.5 \mathrm{~nm}$ thick $\mathrm{MoO}_{3}$ devices. Negative differential resistance (NDR) region was clearly observed in $0.5 \mathrm{~nm}$ thick $\mathrm{MoO}_{3}$ devices with a peak to valley ratio of 6 . A similar NDR behavior was reported in metal NP based organic memory devices ${ }^{3}$ and metal-insulator-metal structures. ${ }^{22} \mathrm{~A}$ charge storage mechanism was proposed by Simmons and Verderber about the NDR behavior ${ }^{22}$ and it was confirmed by Bozano et al. in their metal NP embedded OBDs. ${ }^{3}$ The $\mathrm{MoO}_{3}$ interlayer device showed similar NDR curves of metal NP embedded OBDs and the NDR can be interpreted based on Simmons and Verderber (SV) model. ${ }^{22}$ The SV model explains the NDR behavior of memory devices based on charge trapping and detrapping by a charge trapping center during forward and reverse scans. $\mathrm{MoO}_{3}$ plays a role of charge trapping center in this work and similar $I-V$ curves were obtained. It was reported that NPB formed a CT complex with $\mathrm{MoO}_{3},{ }^{20}$ but DNTPD did not form a CT complex with $\mathrm{MoO}_{3}$ in our experiment, as shown in Fig. 2. Therefore, a CT com-

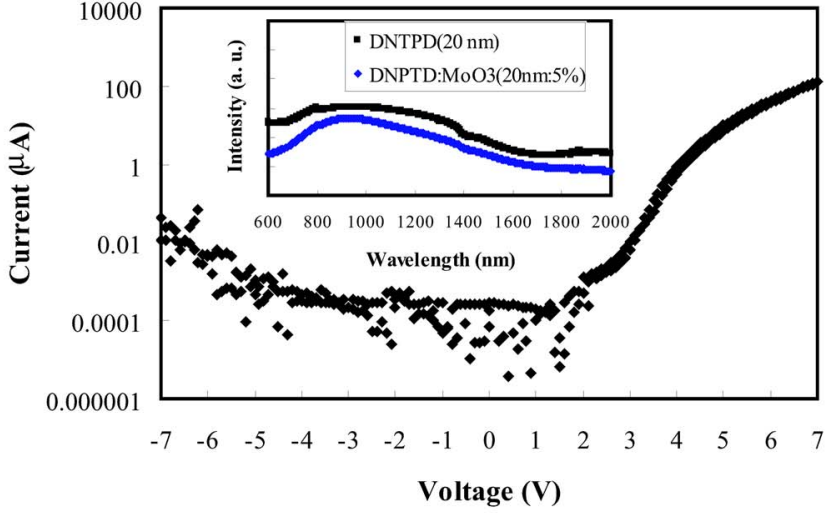

(a)

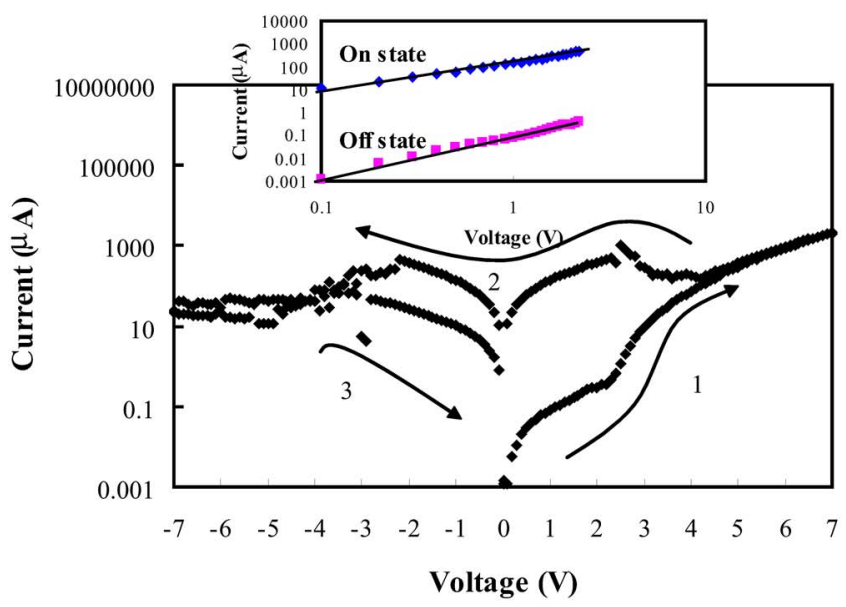

(b)

FIG. 2. (Color online) Current density-voltage curves of organic light emitting memory devices with different $\mathrm{MoO}_{3}$ thickness. Voltage was scanned from 0 to $7 \mathrm{~V}(1), 7$ to $-7 \mathrm{~V}(2)$, and -7 to $0 \mathrm{~V}$ (3). (a) $0 \mathrm{~nm}$ (inset is a ultraviolet-visible-near infrared absorption spectra of DNTPD and $\mathrm{MoO}_{3}$ doped DNTPD). (b) $0.5 \mathrm{~nm}$ [inset is a $\log$ (current)- $\log$ (voltage) plot].

plex of NPB and $\mathrm{MoO}_{3}$ is partially generated at the interface between DNTPD and NPB, while some $\mathrm{MoO}_{3}$ may exist as a free NP dispersed at the interface. The free $\mathrm{MoO}_{3} \mathrm{NP}$ can play a role of charge traps at the interface and the NDR behavior can be induced according to SV model.

The mechanism for charge injection and transport in HCS and the LCS was investigated and log-log plot of current and voltage is shown in inset of Fig. 2. $\log (I)$ and $\log$ $(V)$ showed a linear relationship at HCS and LCS, indicating that a space charge limited current mechanism dominates current injection in $\mathrm{MoO}_{3}$ interlayer devices. ${ }^{19}$ This result implies that $\mathrm{MoO}_{3}$ played a role of charge trap and the space charge formation by charge trapping determined current transport behavior in $\mathrm{MoO}_{3}$ interlayer devices. Some $\mathrm{MoO}_{3}$ NPs, which does not form a CT complex with NPB, may trap charges and induces the space charge limited current behavior.

Switching performances of $0.5 \mathrm{~nm}$ thick $\mathrm{MoO}_{3}$ OLEBDs were evaluated by repeating write-read-erase-read cycles and they were recorded in Fig. 3. Read voltage was $1 \mathrm{~V}$, while write and erase voltage was 4 and $-4 \mathrm{~V}$, respectively. There was little change of the HCS and the LCS current level during multicycle switching test and it was quite stable. Current 


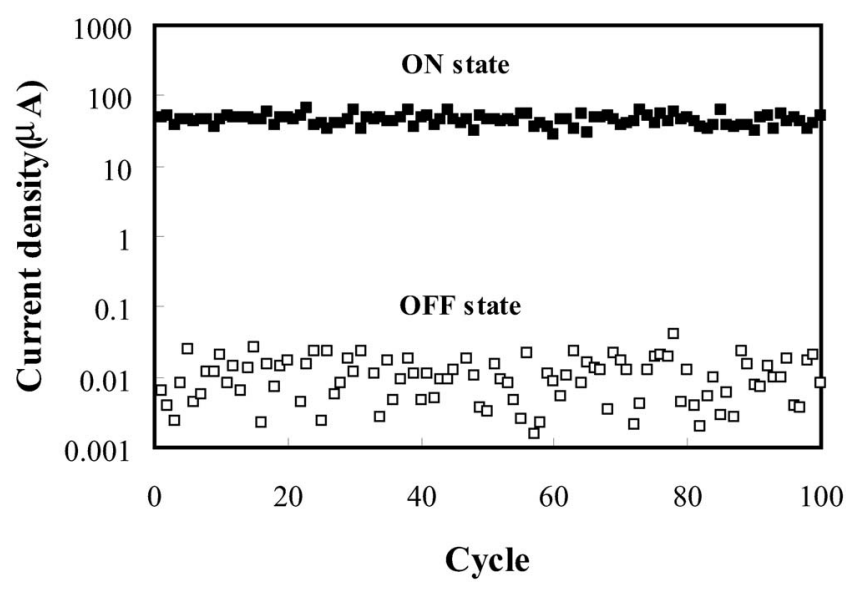

FIG. 3. On-off behavior of organic light emitting memory devices with 0.5 $\mathrm{nm}$ thick $\mathrm{MoO}_{3}$ devices at a write voltage of $4 \mathrm{~V}$, erase voltage of $-4 \mathrm{~V}$, and reading voltage of $1 \mathrm{~V}$.

level was increased by more than 1000 times by applying a write voltage of $4 \mathrm{~V}$ and then it was dropped to LCS by applying an erase voltage of $-4 \mathrm{~V}$.

OLED performances of OLEBDs were also measured according to $\mathrm{MoO}_{3}$ thickness and they are shown in Fig. 4 . $\mathrm{MoO}_{3}$ interlayer OLEBDs showed a much higher current density than standard devices due to p-doping effect of $\mathrm{MoO}_{3}{ }^{20}{ }^{20}$ A high current density was obtained in the $0.5 \mathrm{~nm}$ thick $\mathrm{MoO}_{3}$ interlayer device and driving voltage was $7.2 \mathrm{~V}$ at $1000 \mathrm{~cd} / \mathrm{m}^{2}$. The decrease of a driving voltage in the $\mathrm{MoO}_{3}$ interlayer device can be explained by a $p$-doping effect of $\mathrm{MoO}_{3}$ with aromatic amine materials. An Ohmic contact behavior of $\mathrm{MoO}_{3}$ doped HTL was reported and high conductivity could be observed. ${ }^{23}$ Therefore, $\mathrm{MoO}_{3}$ interlayer can also have a partial $p$-doping effect at the interface between DNTPD and NPB and facilitate hole injection from DNTPD and NPB. The $p$-doping effect is efficient in $0.5 \mathrm{~nm}$ thick $\mathrm{MoO}_{3}$ device because of interfacial contact between $\mathrm{MoO}_{3}$ and NPB, as seen in the TEM data of Fig. 1. There was $0.8 \mathrm{~V}$ decrease of a driving voltage in the $0.5 \mathrm{~nm}$ thick $\mathrm{MoO}_{3}$ device. Therefore, $0.5 \mathrm{~nm}$ thick $\mathrm{MoO}_{3}$ interlayer con-

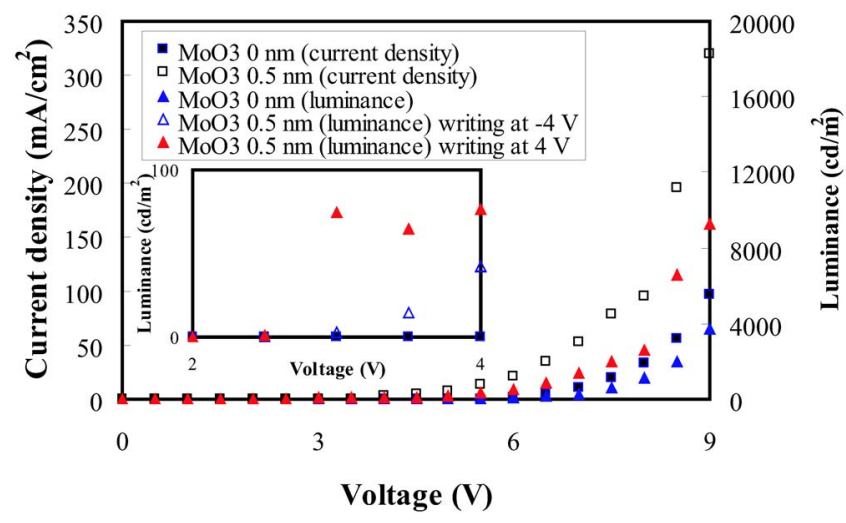

FIG. 4. (Color online) Current density-voltage-luminance curves of organic light emitting memory devices with different $\mathrm{MoO}_{3}$ thickness. Inset is voltage-luminance data of OLEBDs after writing the devices at -4 and $4 \mathrm{~V}$. tributed to the low driving voltage as well as good memory performances in OLEBDs. In addition, different luminances were obtained in OLEBDs depending on the writing voltage of OLEBDs. As can be seen in Fig. 4, luminance of OLEBD after switching on at $4 \mathrm{~V}$ was higher than that of OLEBD before switching from 2.5 to $4 \mathrm{~V}$ and turn-on voltage was lowered from 3.0 to $2.5 \mathrm{~V}$. Therefore, two different luminance states can be obtained by using OLEBD due to bistable memory behavior and it can be expected that selfdriven OLEDs can be developed by optimization and extension of this technology.

In summary, OLEBDs with both OLED and OBD functions could be fabricated by inserting $0.5 \mathrm{~nm}$ thick $\mathrm{MoO}_{3}$ layer between HIL and HTL. $\mathrm{MoO}_{3}$ interlayer was effective to get a low driving voltage in OLEDs by lowering interfacial energy barrier and a high on/off ratio over 1000 could be obtained in OLEBDs with $\mathrm{MoO}_{3}$ interlayer. In addition, two different luminance state could be obtained by changing the writing voltage of OLEBDs and it can be expected that selfdriven OLEDs can be fabricated by extending and further optimizing OLEBD device structures.

${ }^{1}$ L. Li, Q. Ling, S. Lim, Y. Tan, C. Zhu, D. S. H. Chan, E. Kang, and K. Neoh, Org. Electron. 8, 401 (2007).

${ }^{2}$ J. Ouyang, C. Chu, C. R. Szmanda, L. Ma, and Y. Yang, Nat. Mater. 3, 918 (2004).

${ }^{3}$ L. D. Bozano, B. Kean, M. Beinhoff, K. R. Carter, P. M. Rice, and J. C. Scott, Adv. Funct. Mater. 15, 1933 (2005).

${ }^{4}$ Y. Yang, J. Quyang, L. Ma, R. J. Tseng, and C. Chu, Adv. Funct. Mater. 16, 1001 (2006).

${ }^{5}$ R. J. Tseng, J. Huang, J. Quyang, R. B. Kaner, and Y. Yang, Nano Lett. 5, 1077 (2005).

${ }^{6}$ J. Ouyang, C. Chu, D. Sieves, and Y. Yang, Appl. Phys. Lett. 86, 123507 (2005).

${ }^{7}$ A. Prakash, J. Quyang, J. Lin, and Y. Yang, J. Appl. Phys. 100, 054309 (2006)

${ }^{8}$ H. Wang, S. Pigeon, R. Izquierdo, and R. Martel, Appl. Phys. Lett. 89, 183502 (2006).

${ }^{9}$ C. Tu, D. Kwong, and Y. Lai, Appl. Phys. Lett. 89, 252107 (2006).

${ }^{10}$ J. Chen and D. Ma, Appl. Phys. Lett. 87, 023505 (2005).

${ }^{11}$ Y. Lai, C. Tu, D. Kwong, and J. S. Chen, Appl. Phys. Lett. 87, 122101 (2005).

${ }^{12}$ M. Lauters, B. McCarthy, D. Sarid, and G. E. Jabbour, Appl. Phys. Lett. 87, 231105 (2005).

${ }^{13}$ J. Chen, L. Xu, J. Lin, Y. Geng, L. Wang, and D. Ma, Appl. Phys. Lett. 89, 083514 (2006).

${ }^{14}$ A. Kanwal and M. Chhowalla, Appl. Phys. Lett. 89, 203103 (2006).

${ }^{15}$ M. Lauters, B. McCarthy, D. Sarid, and G. E. Jabbour, Appl. Phys. Lett. 89, 013507 (2006).

${ }^{16}$ T. Ouisse and O. Stephan, Org. Electron. 5, 251 (2004).

${ }^{17}$ R. J. Tseng, J. Quyang, C. Chu, J. Huang, and Y. Yang, Appl. Phys. Lett. 88, 123506 (2006).

${ }^{18}$ S. H. Kang, T. Crisp, I. Kymissis, and V. Bulovic, Appl. Phys. Lett. 85, 4666 (2004).

${ }^{19}$ X. Gao, D. Zou, K. Fujita, and T. Tsutsui, Appl. Phys. Lett. 81, 4508 (2002).

${ }^{20}$ H. Ikeda, J. Sakata, M. Hayakawa, T. Aoyama, T. Kawakami, K. Kamata, Y. Iwaki, S. Seo, Y. Noda, R. Nomura, and S. Yamazaki, SID Int. Symp. Digest Tech. Papers 37, 923 (2006).

${ }^{21}$ S. H. Kim, J. Jang, K. S. Yook, and J. Y. Lee (unpublished).

${ }^{22}$ J. G. Simmons and R. R. Verderber, Proc. R. Soc. London, Ser. A 301, 77 (1967).

${ }^{23}$ J. Yun, C. Lee, W. J. Song, and Y. J. Sung, International Meeting on Information Display 7, 691 (2007). 